\title{
Une nouvelle voye pour aller en Parnasse
}

Modernité des vers mesurés à l'antique

Olivier Bettens

\section{OpenEdition}

1 Journals

Édition électronique

URL : https://journals.openedition.org/eve/1005

DOI : 10.4000/eve.1005

ISSN : 2425-1593

Éditeur :

Université de Savoie, Université Jean Moulin - Lyon 3

Référence électronique

Olivier Bettens, "Une nouvelle voye pour aller en Parnasse », Corpus Eve [En ligne], Domaine français, mis en ligne le 18 octobre 2013, consulté le 16 avril 2022. URL : http://journals.openedition.org/eve/ 1005 ; DOl : https://doi.org/10.4000/eve.1005

Ce document a été généré automatiquement le 16 avril 2022

(c) Tous droits réservés 


\section{Une nouvelle voye pour aller en Parnasse}

Modernité des vers mesurés à l'antique

Olivier Bettens

\section{NOTE DE L'AUTEUR}

Cet article fait provisoirement le point d'un travail commencé en 1993, plusieurs fois laissé en l'état pour être ensuite repris. Il tire sa matière d'exposés faits à l'Université de Fribourg, sur l'invitation de Simone de Reyff et au Conservatoire populaire de musique de Genève sur celle de David Chappuis. Je tiens à remercier ces deux professeurs de m'avoir donné l'occasion d'exposer à leurs étudiants mes vues sur un sujet aussi « ésotérique ».

\section{"Une nouvelle voye pour aller en Parnasse »: modernité des vers mesurés à l'antique}

1 Il n'est pas rare que la modernité cherche sa légitimité dans l'ancien, ou plutôt le très ancien. Pour rompre avec un passé récent, vécu comme encombrant, usé, barbare, les avant-gardes convoquent des ancêtres mythiques, se réclament de leur autorité et redonnent vie à des formes oubliées. Les historiens parleront ensuite de "renouveau » ou de « renaissance ».

\section{Le fardeau de la « rythme »}

2 Ouvrant le second livre de sa Deffence sur la question de l'art poétique, Joachim du Bellay identifie sans détour l'un de ces fardeaux légués par l'histoire récente :

Quand à la Rythme, je suy' bien d'opinion, qu'elle soit riche, pour ce qu'elle nous est ce, qu'est la quantité aux Grecz, \& Latins. Et bien que n'ayons cet usaige de Piez 
comme eux, si est-ce, que nous avons un certain nombre de Syllabes en chacun Genre de Poëme, par les quelles comme par Chesnons le vers François lié, \& enchainé est contraint de se rendre en cete etroite prison de Rythme, soubz la garde le plus souvent d'une couppe feminine, facheux, \& rude Gëolier, \& incongnu des autres vulgaires ${ }^{1}$.

3 Cette "rythme ", qu'il prononce vraisemblablement rime, n'est autre qu'un condensé des principes de la métrique syllabique traditionnelle du français. Elle réunit sous son nom ce que nous appelons encore aujourd'hui la rime, à savoir la « consonance » de fin de vers (ou homéotéleute ${ }^{2}$ ) et le rhythmos proprement dit, équivalent grec du latin numerus, c'est-à-dire le nombre des syllabes. La " rythme », qui a des relents de seconde rhétorique, est ici ressentie à la fois comme une contrainte fastidieuse, une "prison ", et comme un pis-aller: puisque la poésie française ne connaît pas la métrique quantitative des anciens, il faudra bien qu'elle s'en contente, au moins provisoirement.

4 Pour dépasser ce sentiment de frustration, les humanistes ont le choix entre deux attitudes : promouvoir l'application au français de la métrique des anciens ou valoriser la « rythme » en lui cherchant des lettres de noblesse.

Qui eust gardé notz Ancestres de varier toutes les parties declinables, d'allonger une syllabe, \& accoursir l'autre : \& en faire des piedz, ou des Mains? Et qui gardera notz successeurs d'observer telles choses, si quelques Scavans, \& non moins ingenieux de cest aage entreprennent de les reduyre en $\mathrm{Art}^{3}$ ?

5 Rien n'empêchait, nous dit du Bellay, les poètes du passé d'adapter le français à la métrique quantitative. Et s'ils ne l'ont pas fait, ceux du futur s'y mettront peut-être. En attendant, il est certainement moins difficile de proclamer que la «rythme», loin d'être une invention gothique, remonte aux anciens Gaulois ${ }^{4}$, ce qui la place, en matière d'antiquité, au moins à égalité avec les pieds des Grecs. Reprise par le préfacier d'Étienne Jodelle, autre poète connu pour avoir composé des vers mesurés, la piste gauloise a l'avantage supplémentaire de bouleverser, à l'avantage du français, la généalogie des langues: comme la langue gauloise et la renommée de ses bardes ont "precedé l'aage des plus vieux Poëtes Grecs, \& par consequent aussi des Latins ${ }^{5}$ », le vernaculaire et la poésie français qui, symboliquement, en sont les héritiers directs gagnent une génération et se retrouvent au même niveau que le grec et le latin.

\section{Compter, mesurer}

6 On ne sait pas jusqu'à quel point les humanistes étaient dupes de leur propre supercherie. Eussent-ils sincèrement adhéré à ce pedigree imaginaire que la «rythme » serait restée grevée d'un important désavantage: dans la hiérarchie des savoirs, l'action de dénombrer ou de compter, à laquelle se rapporte le couple rhythmos / numerus, est supplantée par l'action de mesurer qu'implique le metron / metrum. Même les bardes gaulois n'y peuvent rien : l'arithmétique, première branche du quadrivium médiéval, est plus rudimentaire que la géométrie. Du fait de cette infériorité épistémologique, il restait délicat de revendiquer, pour le vernaculaire français, un statut d'égalité avec les langues antiques tant qu'il n'avait pas donné lieu à une poésie mesurée.

7 Dans l'esprit de certains, la trop grande simplicité de la métrique syllabique française rendait la poésie vulnérable voire corruptible :

Le deuil \& le juste dépit (lecteur) que j'ay eu de voir nostre poësie toute souillee \& abastardie par un tas d'esclaves imitateurs qui se sont impudemment fourrez entre

les plus sçavans d'aujourd'huy, m'a tellement dégousté de nostre ryme, pour la voir 
aussi commune aux indoctes qu'aux doctes, \& ceux la autant authorisez en icelle que ceux cy, que je me suis proposé une nouvelle voye pour aller en Parnasse, non encore frayee que des Grecs \& des Latins, \& qui pour son industrie \& trop plus grande difficulté que celle de la ryme, sera comme j'espere, inaccessible à nos rymasseurs d'aujourd'huy 6 .

L'adoption de la métrique quantitative des Grecs et des Latins apparaissait donc comme une façon d'élever le niveau en barrant la route aux trop mauvais poètes que la « ryme » ne suffisait pas à décourager.

9 La description formelle des vers, ou plutôt des types de vers, fait ressortir de manière on ne peut plus claire l'indigence de la métrique syllabique. Soit le schéma du dimètre iambique tel que le Français Baïf l'a repris des Grecs :

$\mathrm{x}-\mathrm{x}-\mathrm{x}-\cup-$.

10 Ses huit positions sont fortement caractérisées. Le macron (-) exige une syllabe longue, le micron $(\smile)$ une syllabe brève, le X marque une position indifférente, autorisant toute syllabe quelle que soit sa quantité. Diverses substitutions, fondées sur l'équivalence d'une longue et de deux brèves sont en plus possibles. Par comparaison, l'octosyllabe français qui, superficiellement, lui ressemble, fait piètre figure: il se limite à huit positions indifférentes suivies d'une position surnuméraire (x) réservée à une éventuelle syllabe féminine et qui, puisqu'elle ne "compte » pas, pourrait tout aussi bien être omise :

$\mathrm{x}$ x x X x x X x (x).

\section{L'urgence d'un tri}

11 Parmi les poètes contemporains de la Pléiade, Marc-Claude de Buttet est peut-être le premier à s'être essayé aux vers mesurés, et en particulier à la strophe saphique. Cette forme comporte classiquement trois vers "grands saphiques", de onze syllabes chacun :

$-\smile-\mathrm{X}-\smile \cup-\cup--$

dont le dernier est suivi d'un petit vers - on discute s'il s'agit d'un vers à part entière ou d'un « tronçon » métrique qui s'accole au vers précédent ${ }^{7}$ - appelé « adonique » :

$$
-\smile \cup--
$$

En voici un exemple, tiré d'une pièce de circonstance dont on peut situer la composition vers $1550^{8}$, date de la restitution de Boulogne par les Anglais. On le lira tout d'abord sans tenir compte des syllabes mises en évidence :

De la mer ou fut Bellone farouche,

Ou Tytan tumbé tout rouge se couche,

Henri le vangeur de l'Angloise outrance

Revient en France.

Ramenant brave son fort exercite, Non jamais veincu dessous sa conduite,

Ja du beau laurier qui par mort s'achete

Cernant sa teste.

De son absence Paris tant malade,

Le sentant venir de bien loin l'oeillade,

Et lui appreste le triumphe, gloire

De sa victoire.

Par les saints temples, les voutes pendantes

Desja s'allument de lampes ardentes : 
Les prestres qui ja les encensoirs ventent

Haut aux Dieux chantent.

Ja desja lon voit de loin aux campagnes

Flamber les armes, blanchir les ensegnes,

Et d'un trot brave la chevallerie

De Mars cherie'.

13 La particularité de cette tentative est qu'elle est tout entière fondée sur des vers féminins rimés : Buttet admet les avoir «fet expressement tumber par sons feminins, car autrement ils ne povoient avoir grace ». Il n'est de plus pas rare que, à l'intérieur des vers, des positions longues du schéma métrique accueillent des syllabes féminines. Ces deux procédés seront critiqués par Étienne Pasquier pour qui l'e féminin n'est « qu'un demy son que l'on ne peut aucunement rendre long » et, en fin de vers, «n'est point compté pour une syllabe $»^{10}$. Si l'on y regarde de plus près, ces vers fournissent le plus parfait exemple d'une prosodie arbitraire : n'importe quelle syllabe peut y occuper n'importe quelle position. Par exemple, les syllabes comportant une voyelle nasale, ainsi que la première syllabe du mot desja, y sont faites tantôt longues, tantôt brèves : on verra que, chez Baïf, elles n'occupent que des positions longues. Les premières de voûte, de prêtres, qui auraient toutes les raisons d'être longues, occupent des positions brèves, et ainsi de suite. Les syllabes mises en évidence ci-dessus sont celles qui entreraient en contradiction avec la prosodie de Baiff.

En somme, les vers «mesurés » de Buttet ne le sont aucunement. Comme dans les vers traditionnels, les syllabes y apparaissent « sans aucun triage $»^{11}$ : ses grands saphiques ne sont rien de plus que des décasyllabes $5 / / 5$ (parfois appelés taratantaras) féminins admettant la césure dite «lyrique $»^{12}$, ses adoniques des tétrasyllabes tout aussi féminins. La nécessité d'un travail préalable sur le vernaculaire français et sa prosodie n'en ressort donc que plus nettement. Dans l'optique des humanistes, un tel tri prosodique est une entreprise aussi importante et urgente que l'établissement d'un dictionnaire ou celui d'une grammaire. Voici comment le grammairien et rhéteur Ramus, autre tenant de la piste gauloise, l'envisagera en 1572 :

Le moyen ce seroit que les poetes Francois saddonnassent a faire leurs vers non seullement par rithme \& mesure de sons semblables, mais par certaine quantite de syllabes longues \& breves, a la facon des poetes Grecs \& Latins, alors ils apporteroient a la langue Francoyse cest ornement tant necessaire. Car en les oyant, en les lisant, en les observant, comme docteurs \& autheurs de ceste louange, ce qui se faict naturellement, se reduiroit en art, \& par consequence la recherche de laccent seroit aisee. À ceste cause fauldroit supplier aux muses Francoyses dentreprendre ce labeur, non pas pour abolir la rithme, qui est fort plaisante \& delectable, mais affin que leur patrie fust esgallee a la Græce \& a litalie touchant la prosodie en quantite \& accent. Et hardiment le premier gentil esprit, qui remplira ses vers mesures dune bonne \& riche poesie, il sera le premier poete des Francois, comme Homere \& Livius ont este des Grecs \& des Latins, devant lesquels ny avoit ny en Grece, ny en Italie aultre poesie que de rithmes, comme nous avons dict ailleurs ${ }^{13}$.

15 Il n'incombait pas aux grammairiens de s'en charger: de même que, à travers les siècles, c'était par la lecture des poètes que s'était transmise la prosodie du grec et du latin, c'était de même aux poètes français que revenait la tâche prométhéenne de l'établir pour leur langue. 


\section{Baïf Prométhée}

Quelle que soit la manière dont on aborde la question des vers mesurés, c'est toujours la figure de Jean-Antoine de Baïf (1532-1589) qui s'impose. Il y a plusieurs raisons à cela.

Premièrement, Baïf embrasse mieux que quiconque l'idéal néoplatonicien d'une fusion entre poésie et musique. En s'assurant la collaboration, au sein de son académie, d'un compositeur majeur comme Claude Le Jeune, il donne à sa démarche toutes les chances de faire école. De fait, c'est justement dans le champ du lien texte-musique que son influence sera la plus profonde et durable puisqu'elle s'étendra sur plus d'un siècle ${ }^{14}$.

Deuxièmement, il y a le volume et la diversité. En incluant les Etrénes de 1574 et le principal manuscrit autographe de Baif, on dispose de 15129 vers au total ${ }^{15}$, soit l'équivalent de l'Iliade. Ce corpus compte près de 115000 mots consécutifs, qu'il est possible de regrouper en près de 5000 lemmes, ou entrées de dictionnaire, différents. C'est évidemment sans proportion avec le reste de la production en vers mesurés français : Nicolas Rapin, par exemple, le plus prolifique parmi les successeurs de Baiff, n'en a laissé que $1623^{16}$. La diversité des mètres, également, impressionne. Alors que la métrique syllabique traditionnelle se contente par la force des choses d'une grosse dizaine de formes dont trois ou quatre monopolisent l'essentiel de la production, les poètes du XVI ${ }^{\mathrm{e}}$ siècle qui, Baïf hormis, composent des vers mesurés se cantonnent, si l'on en croit l'estimation de Jean Brunel ${ }^{17}$, à une quinzaine de mètres différents ; Baïf, quant à lui, en utilise plus d'une centaine ${ }^{18}$. Mais il n'y a pas de réelle limite : constitués essentiellement de macrons et de microns, les schémas métriques sont abstraits et rien ne s'oppose à ce qu'ils passent tels quels d'une langue à l'autre. Logiquement, tout ce qui s'est réalisé une fois en grec ou en latin peut être importé en français et il serait même possible, à l'infini, de créer de nouveaux schémas. Quoi qu'il en soit, l'accumulation des mètres effectivement présents chez Baïf dépasse de loin tout ce qui s'est fait ailleurs ou en d'autres temps. On serait bien en peine, en particulier, de trouver un poète antique aussi polyvalent!

19 Mais cette prouesse métrique n'est rien en comparaison $d u$ travail que, méthodiquement, Baïf entreprend sur la prosodie. Il sera, troisièmement, le seul à mener à son terme le programme qu'établissait Ramus.

Le vers syllabique traditionnel ne requiert que fort peu de défrichage prosodique préalable. Afin de permettre leur dénombrement, les syllabes doivent être délimitées, ce qui, au prix de quelques artifices, ne pose guère de problème. Il est en plus nécessaire de les répartir en deux groupes : les syllabes féminines d'une part et celles qui ne le sont pas, autrement dit les masculines, de l'autre. Cette catégorisation rudimentaire est tout ce qu'il faut à la métrique pour régir l'élision, la césure et l'entrevers, et donc pour donner lieu, rime mise à part, à des vers syllabiques bien formés. Elle ne requiert même pas que soient formalisées des notions plus grammaticales comme celles de quantité ou d'accent tonique.

21 La pratique du vers mesuré est, on s'en doute, infiniment plus exigeante. Comme les schémas métriques comprennent des positions brèves ( $\cup$ ) et des positions longues (-), il doit être possible de déterminer, à l'avance et pour chaque syllabe de chaque mot de la langue, quel type de position elle est en droit d'occuper : une longue, une brève ou éventuellement les deux. Partant si l'on veut de la friche du "naïf françois », le poète devra, tel un paysagiste, apprendre à identifier les rejets sauvages, à les rabattre et à les tailler jusqu'à obtenir les plantes d'ornement qui constitueront le jardin des vers 
mesurés. Savamment aménagé par les mains de l'homme, ce jardin n'est plus la Nature, mais sa représentation stylisée.

\section{Au jardin des syllabes}

Dans les faits de parole, chaque syllabe a une durée, certes mesurable en millisecondes, mais éminemment variable et non reproductible d'une occurrence et d'un locuteur à l'autre. Ce n'est qu'au niveau, plus abstrait, de la langue que peuvent émerger des oppositions binaires de quantité. En latin, par exemple, deux suffixes fréquents comme -ura (substantifs formés sur des verbes: creatura, natura) et -ula (diminutif: fibula, virgula) se distinguent par leur consonne mais aussi, et peut-être surtout, par le fait que l'u du premier est long et l'u du second bref. On a donc -ūra/-ŭla ${ }^{19}$. Il s'agit d'une opposition phonologique incontestée et incontestable dont les théoriciens antiques rendent compte par les termes de physis / natura. On parlera donc de longueur (ou de quantité) par nature.

\section{Longueur par nature}

23 C'est la longueur par nature qui constitue l'étalon du tri prosodique indispensable à toute métrique quantitative. Comment dès lors la transposer en français? Pour un théoricien comme Jacques de La Taille, il suffirait de reprendre telle quelle celle du latin :

Ce que j'ay dit des prepositions Grecques \& Latines, il en fault autant dire de tous les vocables qui sont descendus, ou qui approchent du Latin en mesme signification, de maniere que Sepulchre, impotent, miserable, sedition, \& cent mille autres auront, mesme quantité que Sepulchrum, Impotens, miserabilis, Seditio. Au moyen de quoy je te r'envoye aux Grammairiens Latins, en tant que nostre langue est conforme à la leur, pour apprendre la quantité de la plus grand part de nos sillabes ${ }^{20}$.

Selon ce principe, on aurait sĕpulchre, impǒtent, mǔsěrable, sēdǔtion et aussi nātūre, virgŭle. Une telle doctrine est bien sûr fort économique pour le théoricien qui, s'appuyant sur la prosodie déjà parfaitement balisée du latin, ne risque pas de se perdre dans la jungle du vernaculaire. Conviendra-t-elle pour autant à la pratique? Théodore de Bèze n'est pas de cet avis :

Il me faut du reste avertir ici les étrangers du très petit nombre de syllabes longues dans la langue française, en regard de l'innombrable multitude des brèves. Et, par conséquent, ils doivent plutôt prendre garde à ne pas allonger les brèves qu'à ne pas raccourcir les longues, principalement là où ils peuvent être induits en erreur par la quantité de la langue latine. Ainsi, par exemple, Natura, Vectura, Fortuna, Persona et autres semblables allongent leur pénultième en latin, alors que les mots français nature, voiture, fortune, personne raccourcissent la même syllabe ${ }^{21}$.

La position de Bèze n'est pas sans fondement linguistique. En français, le suffixe -ure, directement dérivé du -ura latin, existe par exemple sous deux variantes opposables: une longue et une brève. Dans un certain nombre de mots, la contraction, au cours de l'évolution phonétique, de la dernière syllabe du radical avec la première du suffixe s'est soldée par un allongement. C'est ainsi que le latin para(t)uram a donné, en français médiéval, pare-ure où les deux voyelles sises de part et d'autre du tiret, tout d'abord prononcées en hiatus, ont fini par se fondre en une seule longue. Ce phénomène n'a pas eu lieu dans un mot comme pastura > pasture. En français du XVI ${ }^{\mathrm{e}}$ siècle (ou en tout cas dans certaines variétés de français du XVI ${ }^{\mathrm{e}}$ siècle), il persiste donc une opposition de 
quantité entre pastŭre et parūre, natŭre et voitŭre se rattachant, comme le veut Bèze, au premier type. Baïf, c'est certain ${ }^{22}$, percevait de telles oppositions. Si l'on se souvient en plus que, dans pasture, l'amuïssement du s implosif a provoqué un allongement de la voyelle $a$, on comprend que ce n'est pas par hasard que le poète a scandé pātŭrĕ et părūrĕ.

\section{Amorcer le tri}

Imaginons Baïf retroussant ses manches au moment d'entreprendre son travail sur la prosodie: il a devant lui un énorme tas de syllabes pêle-mêle qu'il devra trier en décidant, sur la base de critères encore flous, lesquelles sont longues et lesquelles sont brèves. Il va de soi qu'il commencera par extraire les syllabes les plus manifestement longues, celles qui entrent dans une opposition phonologique de quantité et qu'il identifiera aux syllabes longues par nature de la prosodie grecque ou latine. Elles amorceront le tas des « longues». Ensuite, il cherchera à identifier les syllabes les plus manifestement brèves et il tombera inéluctablement sur les syllabes féminines. Tout comme Pasquier, La Taille leur refuse les positions métriques longues :

Les Syllabes qui ont un E feminin sont tousjours breves, non seullement à la fin des vocables comme, dirĕ fairĕ : mais aussi au commencement \& au milieu, comme děmander, chěval, pauvrěment, rěcěvoir ${ }^{23}$.

Partageant manifestement cet avis, Baïf utilisera les syllabes féminines, ainsi que toutes les autres syllabes dont la voyelle est un $e$ féminin, pour amorcer le tas des «brèves ». Il est maintenant un peu plus avancé : il a, à sa gauche, un petit tas de syllabes longues, à sa droite un assez gros tas de syllabes brèves, mais il reste en face de lui un très gros tas de syllabes non triées. Se replongeant dans ce tas du milieu, il va s'efforcer de le réduire en élaborant des critères qui lui permettront de verser le plus grand nombre possible de syllabes à gauche ou à droite. On comprend déjà que, au fur et à mesure des passes, les syllabes non triées seront de plus en plus « moyennes »: le tri n'en deviendra que plus délicat.

\section{Longueur par position}

Le tas de gauche va pouvoir accueillir d'un seul coup un assez gros contingent de syllabes. À la physis / natura, les théoriciens antiques opposent en effet la thesis / positio : cette position affecte les syllabes fermées, autrement dit celles dont le noyau vocalique est suivi d'au moins une consonne qu'on qualifie d'implosive $e^{24}$. On dit que cette consonne fait position et, de ce fait, les syllabes concernées sont, sans égard à la quantité « naturelle » de leur voyelle, versées sur le tas des longues. Le principe de la longueur par position est fort commode : il n'a pas d'ancrage lexical et son application permet donc de recruter pour ainsi dire mécaniquement un nombre très important de syllabes longues. Alors que la longueur par nature se fonde sur la quantité vocalique, celle par position repose avant tout sur la durée présumée des consonnes implosives, ce qui fait suspecter un artifice: d'un point de vue prosodique, ces deux manières de longueur sont différentes et le choix de les associer à une même catégorie métrique comporte, en grec déjà, une part d'arbitraire. Le latin a néanmoins suivi l'exemple du grec et Baïf, sourd aux protestations de Bèze qui juge non pertinente en français la longueur par position, a emboîté le pas. 


\section{Des syllabes communes}

29 peuvent intéresser Baïf. Si l'on remarque, par exemple, qu'il recourt 244 fois au mot amour et que, à 244 reprises, la première syllabe de ce mot occupe une position métrique brève (ou indifférente) et jamais une position métrique longue, il est difficile de ne pas en conclure qu'il avait tranché en versant cette syllabe, avec probablement un certain nombre d'autres, sur le tas de droite. Sur 51 occurrences du mot soleil, la première syllabe, presque toujours prononcée [su], occupe 51 fois une position métrique brève (ou indifférente), il en va de même de la totalité des 28 occurrences des formes du verbe souloir; la première syllabe des formes du verbe saouler, quant à elle, est 21 fois sur 21 dévolue à une position métrique longue. On retrouve ici, saluée par l'unanimité statistique, une opposition phonologique de quantité que la contraction des deux premières syllabes de $s a(t)$ ullare explique parfaitement. Mais il y a une faille dans le crible : pour les 2 occurrences des formes du verbe soulever, la première syllabe, qui n'est pas phonologiquement longue, occupe dans un cas une position longue et dans l'autre une position brève. Si l'on élargit la recherche aux formes de soutenir qui partage le même préfixe, on constate que la syllabe initiale occupe 8 fois une position longue, 6 fois une position brève et une fois une position indifférente: peut-être la frontière de morphème rend-elle plus tolérable aux oreilles de Baïf un "allongement " qui, à l'intérieur du radical, serait choquant. La même observation peut en tout cas être faite pour le préfixe es-/é-: sur les 22 occurrences des formes du verbe écouter, la première syllabe occupe 17 fois une position métrique brève contre 5 fois une longue.

30 À côté des brèves (comme la première syllabe de soleil ou de souloir) et des longues (comme celle de saouler), il existe donc des syllabes dont le statut prosodique est ambigu. À un moment donné, le tri est devenu délicat pour Baïf et les syllabes trop «moyennes» sont restées définitivement dans le tas du milieu. Le latin et le grec connaissent, quoiqu'en moins grand nombre, de telles syllabes qu'on appelle communes et qui peuvent, selon le gré du poète ou les contraintes auxquelles il est soumis, s'adapter tant aux positions métriques longues qu'aux brèves ${ }^{25}$.

\section{Rôle de l'accent tonique}

31 On sait aujourd'hui que la langue française comporte un accent tonique, et que celui-ci frappe la dernière syllabe non-féminine des mots "pleins $»^{26}$. Il s'agit d'un phénomène qui, quoiqu'il traverse toute l'histoire du français, n'a été reconnu et théorisé qu'au $\mathrm{XIX}^{\mathrm{e}}$ siècle. La Taille, par la force des choses, l'ignore : sous la rubrique accent, il donne une règle confuse qui semble directement transposée des grammaires latines ${ }^{27}$. Baïf, lui aussi, ignore cette notion : s'il utilise bien des marques graphiques correspondant aux trois accents, grave, aigu et circonflexe, du grec, c'est selon une logique qui ne recoupe nullement celle de l'accent tonique telle que nous l'envisageons aujourd'hui pour le français. Cependant, l'accent tonique peut, en français, avoir un effet allongeant sur la syllabe qu'il frappe, effet qui va interférer avec la quantité phonologique et est susceptible d'influencer le tri prosodique. Ainsi, La Taille considère-t-il comme généralement longue la dernière syllabe des mots masculins qui se terminent par une voyelle :

Tous mots terminez en voyelles ont la derniere longue, Ayma, aymé, amy, vaincu. Il ne s'en trouve point en 0 , si ce n'estoient noms propres. Je m'en rapporte à ceux qui 
voudront accourcir les adverbes en $\mathrm{A}$ : à sçavoir de-ja*, pieça*, \&c. Pour le moins tu variras ceux cy, ainsi*, ici*, aussi*, cecy*, nenny*, quasi*. Quant à La Article, \& les Pronoms Ma, Ta, Sa, ils sont brefs, comme aussi sont quelques autres monosyllabes $^{28}$.

Et il ajoute en marge :

Il faut excepter la voyelle feminine. (pâme/Dame, grâce/place, prêche/peche) mais, du fait de sa sensibilité personnelle à l'accent tonique, il semble admettre que l'élément le plus bref de ces paires est, dans l'absolu, déjà trop long pour pouvoir figurer au nombre des syllabes prosodiquement brèves. L'attitude de Baïf face à ces syllabes est plus nuancée : s'il associe, comme on l'a $\mathrm{vu}$, la longueur phonologique à la longueur par nature des théoriciens, il traite par contre en général les pénultièmes qu'elle ne touche pas comme des syllabes communes. Le fait qu'elles soient frappées d'un accent tonique n'est pas, chez lui, une raison suffisante pour les verser sur le tas des longues. Il marque par exemple la première syllabe de pâme d'un accent circonflexe et la place, dans 2 cas sur 2, dans une position longue alors que celle de dame, dans 3 cas sur 7 , occupe une position brève. Il en va de même pour grâce (position longue dans 98 cas sur 98) / place (position brève dans 10 cas sur 16), pour prêche (une seule occurrence dans une position longue avec un accent 
circonflexe)/ pèche (une seule occurrence dans une position brève) et pour fête (position longue dans 25 cas sur 25) / faite (position brève dans 8 cas sur 22).

Serait-il possible de se passer de la notion de syllabe commune et de rendre compte de cette variabilité en recourant à celle d'accent ? Pour Yves-Charles Morin ${ }^{30}$, qui conçoit la syllabe tonique comme une syllabe potentiellement «accentuable " (et non forcément « accentuée »), le fait de rencontrer une fois jŭge droit et une autre juste jūge tiendrait au fait que le mot est accentué dans le second cas mais pas dans le premier. Soit, mais ne serait-ce pas plutôt la métrique qui dicte le choix de Baïf ? Jūgĕ drōit est impossible dans un mètre dactylique où les positions brèves vont toujours par deux et, dans le second exemple (Juste jūgě sur nous), la versification trochaïque "pure» interdit, indépendamment de toute considération accentuelle, que deux syllabes brèves se succèdent : jŭgĕ serait donc, à moins d'une substitution qui affecterait le nombre des syllabes du vers, prohibé. Et comment justifier que, dans Quand Dieu se lèv(e)ra, jŭgĕ pour sauver, le même mot qui était "accentué » devant la préposition sur ne le soit pas devant pour? Peut-on encore appeler accent un «allongement» facultatif qui semble indépendant de la syntaxe mais obéit aux contraintes du mètre et qui, par surcroît, n'est nullement limité aux syllabes toniques puisqu'il peut, comme on l'a vu, toucher de simples préfixes? La notion de syllabe commune rend décidément mieux compte d'une telle réalité.

Très occasionnellement - c'est loin d'être la règle - la longueur phonologique portant sur une syllabe tonique peut s'estomper chez Baïf lorsque la syllabe correspondante devient prétonique. On a, par exemple, bête, dont la première syllabe occupe 20 fois sur 20 une position longue alors que celle de bétail occupe 7 fois sur 22 une position brève, et devrait donc être considérée comme une syllabe commune. Le même phénomène s'observe pour la première syllabe de maitre ( 11 fois sur 11 dans une position longue) / maîtresse (3 fois sur 4 dans une position brève).

\section{Des oppositions non rentables?}

Baïf n'a pas pu tirer parti de la totalité des oppositions phonologiques de quantité qu'il perçoit. Les troisièmes personnes des imparfaits et conditionnels, par exemple, se terminent en -oit (bref) au singulier et en -oient (long) au pluriel. Baïf note assez consciencieusement cette opposition en coiffant les pluriels d'un accent circonflexe et les singuliers d'un accent grave, mais toutes ces finales, indépendamment de leur quantité phonologique, occupent sans exception des positions longues dans ses vers. S'il avait, lors d'une première passe de son tri, pu isoler les pluriels en les versant sur le tas des longues, il a, dans une passe ultérieure, fait de même avec les singuliers parce qu'il considère leur noyau vocalique comme une diphtongue et que, selon la théorie antique, les diphtongues sont longues par définition. La différenciation graphique qui rend compte de cette opposition phonologique, si elle perd tout rendement métrique, garde en revanche une valeur diacritique.

Dans le même ordre d'idées, il existe, pour les mots en -é (notamment les participes passés) une opposition phonologique de quantité entre les masculins et les féminins (ée dans la graphie usuelle). Baiff en rend compte au moyen d'un circonflexe sur les féminins, mais cette opposition n'a pas plus de rendement métrique que la précédente puisque, comme on l'a vu, les masculins en -é occupent déjà systématiquement des positions longues dans ses vers. L'opposition de quantité qui pourrait éventuellement exister entre singulier (-é) et pluriel (-és) n'est, pour la même raison, pas non plus 
exploitée métriquement. Par contre, les finales des infinitifs en -er se trouvent perdre leur longueur par position lorsque le mot suivant commence par une voyelle avec laquelle le $-r$ final est resyllabé. Lorsque cela se produit, Baïf leur réserve presque systématiquement une position brève. Pour cette voyelle caractérisant les verbes du premier groupe, la ligne de démarcation résultant du tri de Baiff passe donc étonnamment entre l'infinitif et les autres formes.

\section{L'exacte écriture conforme au parler}

Baïf en est là. Des mois, des années durant peut-être, il a trié, aussi loin que ses forces et ses sens le lui permettaient, mais il n'a pas encore écrit une seule ligne. Pour rendre compte de son travail, il peut décider de rédiger un traité théorique, de compiler un dictionnaire prosodique du vernaculaire, ou de composer des vers. On sait quel a été son choix.

41 Il est périlleux d'aborder les aspects graphiques car c'est sa graphie qui, sans doute, a attiré sur Baïf Prométhée le châtiment éternel. Les premières critiques, en effet, ne visent pas tant sa prosodie, ou sa métrique dont chacun s'accorde à reconnaître le caractère docte, que la "rudesse du langage » - on lui reproche probablement les néologismes et les distorsions syntaxiques auxquels il recourt pour traduire les anciens de manière littérale - et, «sur tout sa bizarre et pretentieuse façon d'écrire où il faloit deviner plutost que lire et occuper son esprit si entierement a dechifrer ses figures de caracteres qu'on ne pouvoit penser à ce qu'il vouloit dire $»^{31}$.

La première question qu'on posera est celle-ci : la graphie de Baiff est-elle, comme cela serait le cas dans un calligramme, constitutive de sa poésie? Autrement dit, ses vers mesurés perdraient-il quelque chose de leur forme et de leur substance si leur graphie était modifiée, standardisée, modernisée ? À quoi il faut, résolument, répondre par la négative. La graphie de Baï est bien sûr pour nous une mine de renseignements inestimable et un fascinant objet d'étude. Mais autant nous, lecteurs béotiens, en avons besoin, autant le poème en soi peut s'en passer. Un poète aguerri, après avoir intériorisé le système de Baiff, pourrait fort bien produire des vers mesurés qui, sans se conformer à ses principes graphiques, en respecteraient scrupuleusement la prosodie et dont le mètre serait perceptible à un lecteur qui aurait développé les mêmes compétences. C'est précisément le cas de Jodelle qui livre, en 1567 déjà, comme pièce liminaire des Météores de Baîf, cette élégie "à la France » en dix-huit distiques élégiaques, dont la graphie est on ne peut plus ordinaire et dans laquelle, abstraction faite de toute considération graphique, on cherchera longtemps la moindre entorse à la prosodie de Baîf :

Sur ce que tourne le ciel, \& sur ce que close dedans luy

Forme la Terre encor, l'Onde, le Vuide le Feu :

Combien voy-je en toy sans cesse se naistre de terreurs.

Et sans cesse en toy, FRANCE, se naistre d'abus ${ }^{32}$ ?

43 À fin de comparaison, on rappelle que, dans les strophes saphiques de Buttet, plus du tiers des syllabes entrent en contradiction frontale avec cette même prosodie, contradiction qui ne serait en rien résolue par la translittération des vers de Buttet dans la graphie de Baïf. Voilà qui montre que, dès avant la création officielle de l'Académie (1570), le savoir-faire prosodique compilé par Baïf avait pu s'abstraire de sa graphie et diffuser au sein d'un cercle restreint dont Jodelle faisait certainement partie et dont Buttet était manifestement exclu. À mi-chemin, les asclépiades mineurs (- - - 
$\cup \cup--\cup \cup-\cup \mathrm{x}$ ) produits par Charles Toutain en 1556, et dont la graphie n'a rien d'extravagant, font déjà montre d'un réel souci prosodique :

Avertir je me veus quand tuer Atropos

Bien tôt viendra ce corps, et que tes ïeus rians

Sans plus rire seront, Clorine, larmoians

Ton Toutain abatu sous le chetif repos ${ }^{33}$ :

Respect de la brièveté des $e$ féminins, de la longueur par position et, pour autant qu'on puisse en juger, sensibilité à la longueur phonologique caractérisent cette tentative. Seules une vingtaine de syllabes sur soixante vers $(3 \%)$ ne concordent pas avec les choix, bien sûr ultérieurs, de Baïf.

Mais alors, poursuivra-t-on, pourquoi Baïf s'est-il obstiné à défendre une graphie si peu indispensable alors même qu'elle était critiquée de toutes parts et nuisait à la réception de son œuvre ? Parce que, probablement, elle était un outil de travail qui l'avait aidé à organiser sa pensée, à consigner ses choix prosodiques, à formaliser son système et à forger ses vers. Il s'y était attaché et il espérait, à tort, qu'elle serait suffisante pour diffuser, au-delà du premier cercle, le « mode d'emploi » de sa poésie mesurée.

On le sait ${ }^{34}$, Baïf est tout sauf un réformateur de l'orthographe à l'image de Meigret ou de Ramus. Il se défend du reste d'avoir jamais voulu faire changer l'écriture officielle, celle des "commis ", et ne revendique sa graphie que pour ses propres vers mesurés ${ }^{35}$. Mais si elle est riche en informations, cette graphie n'en est pas moins difficile d'interprétation - beaucoup plus que ne le serait une graphie unilatéralement phonétique - car s'y entrecroisent et s'y superposent des éléments phonétiques, des éléments prosodiques et des éléments métriques ${ }^{36}$.

\section{Des consonnes fictives}

On peut avoir l'impression, à première vue, que Baïf ne note que les consonnes qu'il prononce et cela est le plus souvent vrai si l'on excepte les consonnes finales. Il renonce en tout cas aux nombreuses consonnes que Bèze qualifie de "quiescentes " parce qu'il les voit mais ne les entend pas $^{37}$. Mais que penser lorsqu'on tombe sur la graphie: rizzê' [risée]. Baïf entend-il vraiment deux z consécutifs ? C'est fort peu vraisemblable. On est en fait en présence d'un pur artifice graphique qui mime un mécanisme prosodique - la longueur par position - dans le seul but d'apporter une information sur le schéma métrique : dans ce vers particulier, la première syllabe du mot risée occupe une position longue. Comme la même syllabe, dépourvue de cet artifice, peut apparaître ailleurs dans une position métrique brève, on conclut qu'elle appartient au groupe des communes. Au lieu de redoubler la consonne, Baïf aurait aussi bien pu la surmonter d'un macron, ou alors ne rien faire et laisser le lecteur deviner la valeur métrique de la position correspondante.

\section{Les consonnes finales}

$\mathrm{Au}$ temps de Baif, la prononciation des consonnes finales, en particulier dans la diction du vers, est à géométrie variable : systématiquement articulées devant voyelle initiale, elles tendent à tomber devant consonne initiale; leur statut phonétique en fin de vers (et, s'il y a lieu, à la césure) est beaucoup plus incertain ${ }^{38}$. Même non prononcées, elles gardent, aux yeux du lecteur, une valeur diacritique qui aide, par exemple, à la distinction des singuliers et des pluriels, ce qui explique qu'elles aient mis en difficulté 
tous les réformateurs orthographiques du XVI siècle, déchirés qu'ils étaient entre les exigences contradictoires de la conformité à la prononciation et de la lisibilité grammaticale.

Elles ont bien sûr aussi préoccupé Baïf qui a essayé, quoique de manière inconstante, de substituer un tiret à certaines consonnes finales qu'il ne prononçait pas. Dans le cas des vers mesurés, elles posent un problème supplémentaire: devant consonne initiale, c'est-à-dire dans une situation où, en général, on ne les entend pas, elles vont, si elles sont maintenues dans la graphie, faire fictivement position.

Après quelques hésitations dans le psautier $\mathrm{A}$, Baïf décidera (dès les Etrénes et le psautier B de 1573) de les ignorer totalement dans les syllabes féminines, par principe exclues des positions métriques longues mais, par contre, d'en tenir compte dans les syllabes masculines. Ainsi, lorsqu'il veut par exemple placer le mot tout dans une position métrique brève, il est obligé de réunir graphiquement deux mots et d'écrire : ţle-peple (tout le peuple). Si, conformément au découpage en mots communément admis, il écrit: tot le monde (tout le monde) la même syllabe ne peut plus occuper qu'une position longue. Comme dans risée, une fiction prosodique fournit au lecteur une information qui n'a trait qu'au schéma métrique.

Par contre, si le mot suivant commence par une voyelle, Baïf fait face à un vrai choix prosodique car la consonne finale du mot est resyllabée avec la voyelle suivante et cesse par conséquent de faire position. Dans ce cas, il place tout dans une position brève (ou indifférente) dans 193 cas sur 193, ce qui indique qu'il avait préalablement versé cette syllabe sur le tas des brèves. Dans le cas du féminin toute(s), la même première syllabe n'occupe que 3 fois sur 206 occurrences une position longue, ce qui est probablement négligeable. Au pluriel, par contre, tous devant voyelle occupe 5 fois une position brève contre 22 fois une position longue, ce qui montre que Baïf accordait plus de poids à la forme du pluriel puisqu'il l'avait au moins rangée parmi les communes ${ }^{39}$. En étendant la recherche aux pronoms nous et vous devant voyelle, on obtient au total 95 positions brèves contre 46 longues, ce qui semble bien confirmer que le groupe des monosyllabes en -ous à faible contenu lexical - cette catégorisation vaut ce qu'elle vaut - a été versé dans les syllabes communes. Le fait que les pronoms nous et vous puissent-être postposés, et donc en principe frappés par l'accent, n'est en lui-même pas déterminant quant à leur valeur métrique : on trouve aussi bien: Porr n̋̌z il ę̂t (Pour nous, il est) que Vakes nōs usset kðvęrs (Vagues nous eussent couverts).

Pour les polysyllabes, le choix de départ est le même, mais la réponse de Baï sera sensiblement différente. Sur près de 400 occurrences de mots en $-\mathrm{s} / \mathrm{z}$ après voyelle ${ }^{40} \mathrm{et}$ devant voyelle initiale, la syllabe finale n'occupe une position brève que dans 3 cas qui sont tous des noms propres gréco-latins (Venus, Pallas, Bacchus). Baif avait donc, à un moment ou un autre de son tri, versé ces syllabes au nombre des longues.

Dans la même situation, - $d / t$ est loin d'avoir le même effet allongeant que -s/z puisque, sur 57 occurrences au total, seules 4 occupent une position longue. Ces syllabes ont donc au mieux été triées comme des communes, ce qui permet à Baiff de tirer un parti métrique de l'opposition phonologique de quantité qu'il perçoit, par exemple, entre fut (parfait) et fût (imparfait du subjonctif) ou entre les troisièmes personnes des verbes formés sur le modèle de fleurir dont la conjugaison française a pu garder, au présent, une trace du suffixe inchoatif latin (-sc-) et dont la dernière voyelle est par conséquent longue à la troisième personne du présent (fleurit) et brève à celle du parfait (fleuritt). 
Pour -r, toujours dans la même situation, on a 239 positions brèves contre 18 longues : les syllabes finales correspondantes sont donc au mieux communes.

51 En définitive, les consonnes finales semblent bien exercer, sur la voyelle précédente, un effet qui influence le tri prosodique de Baïf et que La Taille résume assez bien en considérant comme abrégeantes ${ }^{41}$ les consonnes finales $-b,-c,-f,-q,-l,-r,-t,-p,-g,-d$ et comme allongeantes $-s$ et $-z$, noms propres intraduisibles exceptés (Phebus, Jesus, Pallas, Atlas).

\section{Marques prosodiques et marques métriques}

Avant d'aborder les voyelles, il faut parler brièvement de quelques-unes des marques dont Baiff surmonte certaines d'entre elles. Fondamentalement, elles sont de deux types: métrique et prosodique. Les marques prosodiques (essentiellement les accents circonflexe, grave et aigu) expriment un jugement de portée générale sur la voyelle qu'elles surmontent. Dans le cas du circonflexe, la voyelle en question est longue; avec les deux autres accents, elle ne l'est pas. Noté une fois, l'accent vaut pour toutes les occurrences de la syllabe concernée. Baïf est en effet assez peu systématique dans sa manière de noter les accents, qu'il omet souvent. Par contre il est très cohérent et ne notera en principe pas, sur la même syllabe, tantôt un accent circonflexe et tantôt un accent grave. Le circonflexe signale presque toujours, chez lui, la longueur phonologique. Par exemple, l'accent circonflexe ne coiffe qu'une seule fois (sur les 56 occurrences que compte le corpus) la première syllabe du mot dôser [douceur]. Pourtant, 56 fois, cette syllabe occupe une position métrique longue. Un seul circonflexe vaut donc pour 56 occurrences. On sait par ailleurs que la voyelle correspondante est phonologiquement longue puisqu'elle résulte de la vélarisation du $l$ implosif de dulcorem.

Les marques métriques (essentiellement le macron et le micron) donnent une information qui ne porte pas sur la prosodie des syllabes correspondantes mais sur la valeur métrique de la position qu'elles occupent dans un vers particulier: le macron indique une position longue et le micron une position brève. Pour la première syllabe de douceur, 14 occurrences sur 56 sont surmontées d'un macron. Il serait au demeurant parfaitement possible, même si cela ne se rencontre pas pour ce mot, qu'un macron et un accent circonflexe coexistent au-dessus de la même syllabe puisque l'information donnée par ces deux marques n'est pas la même et n'a pas la même portée. Par comparaison, la première syllabe de douleur, qui se trouve en opposition phonologique de quantité avec celle de douceur est surmontée d'un micron dans 10 occurrences sur 54. Dans la totalité des cas, elle occupe une position brève.

Il est aussi parfaitement normal qu'une même syllabe - on la considérera par définition comme commune - soit surmontée d'un macron dans certaines occurrences et d'un micron dans certaines autres. Il en va ainsi de la dernière syllabe de voici, dont 14 des 33 occurrences sont surmontées d'un macron et 13 surmontées d'un micron, le lecteur étant laissé livré à lui-même dans les 6 occurrences restantes.

\section{Voyelles et fictions}

Il saute aux yeux que la graphie de Baif, imitant en cela celle du grec, a recours, pour les voyelles $e$ et $o$, à plusieurs variantes concurrentes; c'est ce qui l'a longtemps fait considérer comme le tenant d'une orthographe phonétique, au même titre que Meigret, 
Peletier ou Ramus. La réalité se révèle bien plus complexe car, une fois de plus, phonétique, prosodie et métrique sont fortement enchevêtrées.

Le caractère e, qui figure chez lui $e$ féminin, est le seul à être exempt de toute ambiguïté. On a une valeur phonétique bien identifiée, [ə], qui rend prosodiquement brèves toutes les syllabes dont elle est la voyelle, ces syllabes ne pouvant, de ce fait, occuper que des positions métriques elles aussi brèves.

À l'opposé, les deux o que connaît la graphie de Baïf suscitent de nombreuses interrogations. On s'aperçoit vite, par exemple, qu'il n'existe aucune correspondance systématique entre les deux timbres phonétiques, fermé et ouvert, que connaît aujourd'hui le français standard, à savoir [o] et [o], et les deux graphies o (omicron) et $\infty$ (oméga) dont se sert Baïf. La situation n'est pas moins complexe du point de vue prosodique. Rien qu'en initiale atone, quatre cas de figure peuvent se présenter :

- $\infty$ surmonté (inconstamment) d'un circonflexe : autel, aubade. On sait alors qu'on a affaire à une voyelle phonologiquement longue (vélarisation d'un l), au nombre de celles que Baiff, lors de la première passe de son tri prosodique, a versées sur le tas des longues, mais c'est le circonflexe et non la variante graphique $\infty$ qui est vecteur de cette information. On sait aussi qu'on ne rencontrera jamais cette syllabe dans une position métrique brève.

- $\infty$ sans circonflexe : automne, auguste, audace, aurore, augure, auteur. La voyelle n'est pas phonologiquement longue, mais le fait que Baiff semble réserver à ces syllabes initiales la variante $\infty$ pourrait indiquer qu'il avait, lors d'une passe ultérieure, décidé, pour une raison qui lui appartient, de les verser aussi parmi les longues.

- alternance de o et de $\infty$ : oreille, obéir. Baïf n'a pas pu trancher. Il considère, faute de mieux, ces syllabes comme communes. Avec orage (un seul $\infty$ contre $15 \mathrm{o}$ ), on commence à se demander si la seule exception rend vraiment compte du tri prosodique de Baïf ou s'il ne s'agit pas plutôt d'une licence isolée, qu'une contrainte métrique pourrait justifier.

- o exclusivement : océan, odeur, Olympe. Si vraiment Baïf s'interdit la variante $\infty$ pour ces mots (il y a relativement peu d'occurrences et la distribution observée pourrait à la rigueur être due au hasard), cela signifierait qu'il les a versées parmi les brèves.

Cet imbroglio graphico-prosodique se résout comme par enchantement au niveau métrique : la variante o se trouve exclue des positions longues; la variante $\infty$ se trouve quant à elle exclue des positions brèves. Le lecteur d'un vers mesuré, par exemple un compositeur qui le met en musique, peut s'économiser tout raisonnement prosodique : il sait que, en syllabe ouverte, $\infty$ doit être scandé long et o doit être scandé bref. Il n'a plus qu'à appliquer la recette. En résumé, l'opposition o/o chez Baif peut être considérée comme une fiction graphique sans signification phonétique évidente et prenant l'apparence d'une opposition prosodique dans le seul but d'expliciter la métrique des vers.

Quant à l'opposition e/ę elle fonctionne d'une manière analogue, mais plus complexe encore. Comme la place du $e$ «bref » est prise par e (féminin), Baïf qualifiera la variante e de « commune » et la variante $\mathrm{E}_{\boldsymbol{c}}$ de « longue » ${ }^{42}$, ce qui ne préjuge en rien de la valeur prosodique réelle des syllabes qui les contiendront. La valeur phonétique de ces deux variantes n'est pas totalement arbitraire. Lorsqu'elles apparaissent en syllabe fermée, autrement dit dans une syllabe longue par position, Baïf attribue au son [e] (e fermé) la variante e et au son $[\varepsilon]$ ( $e$ ouvert) la variante $E_{c}^{43}$. C'est ainsi qu'on peut lire : fierté, 
mais pubęrte là où l'on attend effectivement [fjerte] et [pybsrte], et aussi, en syllabe finale : réverer (révérer), mais keręt (guéret).

Dans ce dernier cas, il arrive, si le mot suivant commence par une voyelle, que la consonne finale soit resyllabée avec celle-ci et cesse de faire position. Baïf se trouve alors comme prisonnier de la graphie choisie et semble contraint de réserver la dernière syllabe de guéret à des positions métriques longues, ce qui l'empêche d'exploiter métriquement l'opposition phonologique de quantité qui existe entre guéret et forêt. Inversement, dans le cas des infinitifs en -er, il se sent presque obligé de placer leur dernière syllabe dans une position métrique brève et crée par là-même la curieuse opposition entre le participe passé (révéré) et l'infinitif (révérer) dont il a déjà été question ${ }^{44}$.

Comme pour les o, la vraie opposition phonologique de quantité, celle qui existe entre fête et faite, est signalée occasionnellement, non pas par la graphie de la voyelle, mais par l'accent circonflexe. On a donc: fệte qui s'oppose à fęte/féte où la voyelle choisie informe, comme le ferait un macron ou un micron, sur le schéma métrique d'un vers donné (la valeur prosodique est de toute façon commune). Cette alternance graphique $\mathrm{e} /$ ę touche fréquemment les pénultièmes toniques non longues des mots féminins, ou les syllabes finales des mots en -l. Le mot père(s), par exemple, est graphié 27 fois avec é dans une position brève et 21 fois avec $\mathrm{E}_{c}$ dans une position longue ${ }^{45}$.

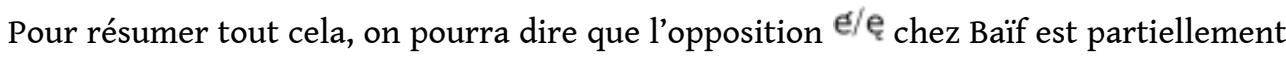
fondée du point de vue phonétique, qu'elle ne fait que mimer sans la recouper une opposition prosodique et qu'elle oriente partiellement le lecteur quant au schéma métrique du vers : dans tous les cas, Ę indique une position longue ; quant à e, en finale absolue ou devant -s/z finaux, il désigne un $e$ fermé ([e]) dans une position longue, en pénultième tonique ouverte ou devant $-\mathrm{r} / \mathrm{l}$ finaux suivis de voyelle initiale un $e$ de timbre indéterminé presque toujours en position brève.

\section{L'ïambe dru je scay rebattre}

Comment un individu d'hier ou d'aujourd'hui, musicien ou non musicien, plus ou moins au fait de la poésie gréco-latine et de la langue française de la Renaissance, peut-il recevoir et ressentir rythmiquement les vers mesurés de Baïf et de ses contemporains? Plusieurs niveaux de lecture se rencontrent.

\section{Un vers libre}

60 Le degré zéro de leur réception consiste à lire les vers mesurés comme des vers syllabiques. Vus sous cet angle, ils apparaissent anarchiques et peuvent, comme le relève Claude-Gilbert Dubois ${ }^{46}$, donner "l'impression d'une libération, verlainienne avant la lettre, de la poésie par la disparition ou l'érosion de la contrainte rimique, et l'introduction de vers impairs. Mais, nuance-t-il, en fait la contrainte est multipliée par l'introduction de schémas calqués sur la poésie antique dans une langue qui les intègre mal ». Le jugement tombe un peu rapidement, ce d'autant plus que son auteur donne du fameux distique de Jodelle pour Olivier de Magny une scansion erronée ${ }^{47}$ !

$61 \mathrm{Au}$ début du $\mathrm{XX}^{\mathrm{e}}$ siècle, André Beaunier avait déjà rapproché le vers mesuré du vers libre des Symbolistes : 
Et c'est en cela que se trompèrent les chimériques réformateurs, tels que Baiff, qui prétendirent constituer une prosodie française à l'imitation de la prosodie latine [...] Aussi, comme je l'indiquais, le français ne se prête-t-il pas à la fabrication de dactyles ou de spondées absolus. Mais les différences de longueur, très nuancées, très variées que présentent les mots d'une phrase française produisent des rythmes très complexes et sollicitent donc une métrique très souple, qui précisément est celle du vers libre ${ }^{48}$.

Dans un jardin laissé à l'abandon, la Nature reprend ses droits...

\section{Un vers pour la musique} mesurés de Baïf, «à la saulse de la musique que leur donna Claudin Le Jeune furent agreables, mais prononcez sans cette ayde furent trouvez fades \& fascheux, surtout pour ce qu'il [Baïf] donnoit au François une dure construction latine $»^{49}$. Pour Jean Vignes $^{50}$, le projet de vers mesurés de Baif ne trouve vraiment son sens «que dans la volonté du poète d'unir plus intimement poésie et musique». Et, s'appuyant sur le travail de Pierre Bonniffet ${ }^{51}$, il conclut que le système graphique de Baïf « vise moins à matérialiser la réalité phonétique ambiante qu'à imposer un rythme vocal aux compositeurs, aux chanteurs, éventuellement aux lecteurs ».

63

rend possible une attitude globalement bienveillante à l'égard de l'apport de Baïf, cette vision présente l'inconvénient d'esquiver complètement la question de la prosodie. Selon Vignes, "la question de savoir si le français parlé présente ou présentait naturellement des longues et des brèves n'est pas directement pertinente pour juger du projet de Baif ", ce qui a pour effet de ravaler le patient et minutieux travail de ce dernier sur la langue au même niveau que la non-prosodie de Buttet. On ne célèbre pas tant l'union de la poésie et de la musique que la prise de pouvoir du poète, proclamé «maître absolu du rythme de son texte », sur le musicien (et sur la langue). C'est somme toute assez peu platonicien.

\section{Une langue insuffisante}

Pour pouvoir prendre racine dans une langue donnée, toute métrique doit y trouver un terreau prosodique favorable. Autrement dit, aux catégories métriques prédéterminées doivent pouvoir correspondre des catégories prosodiques présentant un certain degré d'isomorphisme avec elles et, si possible, pertinentes du point de vue phonologique. On admet par exemple que le grec et le latin n'auraient jamais pu développer une métrique quantitative si leur phonologie n'avait pas, au départ, été « sensible à la quantité ». Sur

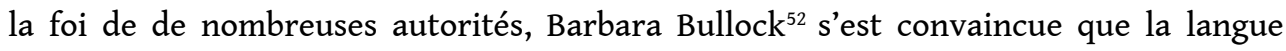
française de la Renaissance est «insensible à la quantité », autrement dit qu'elle ne connaît pas d'oppositions de durée distinctives. De plus, elle travaille sur un minuscule échantillon de trimètres iambiques tiré des Etrénes, dont l'imprimeur a fait sauter la plupart des accents circonflexes que Baif s'astreint à noter dans son manuscrit. Les indices les plus précieux de la présence d'oppositions phonologiques de quantité dans la langue de Baif lui restent donc inaccessibles.

Logiquement, de telles prémisses ne devraient pas lui permettre de conclure grandchose. En plus de relever le respect de la longueur par position, elle postule une coïncidence systématique des ictus métriques avec des syllabes toniques et elle en conclut à une métrique "largement accentuelle ", mais elle a dû pour cela modifier le 
schéma métrique à sa guise ${ }^{53}$. Comme d'autres avant elle, elle ne parvient pas à démystifier les fictions que représentent les alternances $o / \infty$ et $\mathrm{E} / \mathrm{e}$ dans le système graphique de Baï.

Certains auteurs considèrent la langue française comme insuffisamment accentuée pour bien se prêter aux vers mesurés. C'est le cas d'Édith Weber pour qui «la langue allemande, plus accentuée que la langue française, se prête moins mal au traitement prosodique quantitatif que la langue française du XVI ${ }^{\mathrm{e}}$ siècle, arbitrairement "mesurée à l'antique" $\aleph^{54}$. Autrement dit, comme la langue française n'est pas quantitative, il faut que Baïf ait fondé sa métrique sur l'accent, mais comme l'accent n'y est pas suffisamment perceptible, il aurait mieux fait de choisir une autre langue.

Et pourtant... Si, parmi les «grandes » langues d'Europe occidentale, il y en a une qui était, à la Renaissance, équipée pour accueillir une métrique quantitative, c'est bien le français. Comme le relève très justement Morin $^{55}$, ce n'est pas par défaut de quantité de la langue française (ni du reste par défaut d'accent) que Baïf n'a pas réussi à imposer durablement ses vers mesurés, mais par manque d'un consensus culturel. En comparaison, l'ancrage phonologique de la rime n'était en lui-même guère plus solide, mais il s'agissait d'une institution culturelle forte d'une tradition de plusieurs siècles ${ }^{56}$ et qui, de ce fait, pouvait résister à toutes les attaques.

\section{Un péché contre la langue}

Selon Georges Lote, le péché mortel de Baif serait au contraire d'avoir négligé l'accent tonique :

Ce qu'il y a de plus grave, c'est que Baïf n'a aucune idée de ce que peut et doit être un rythme. Visiblement il travaille sur des syllabes inertes, dont la déclamation de ses contemporains ne lui a pas enseigné la valeur. Il se figure que des successions de longues sont capables de satisfaire l'oreille, et il n'a nul souci de l'accent pour les établiir ${ }^{57}$.

Il est en effet parfaitement possible qu'un schéma métrique compte des suites de plusieurs longues. Un dimètre anapestique peut par exemple se décliner sous la forme de quatre spondées, soit huit longues consécutives, ce qui arrive une fois dans

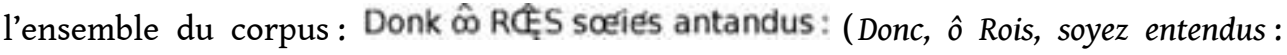
Psautier A, ps. 2, vers 24).

Il ne s'agit peut-être pas du meilleur vers qu'ait composé Baïf, mais en quoi serait-il fondamentalement moins rythmé que n'importe lequel des millions d'octosyllabes qu'a produits la métrique syllabique traditionnelle ? Lote s'explique ainsi :

Il lui [Baïf] aurait suffi de comprendre que les mètres des Anciens, quantitativement irréalisables en français, pouvaient y trouver leur équivalent grâce au jeu normal des toniques et des atones. Mais c'est cela justement qui a échappé à son oreille ${ }^{58}$

69 Comme les oppositions de quantité échappent à l'oreille de Lote, il propose de recourir à d'autres catégories prosodiques - les toniques et les atones - pour occuper les positions métriques longues et brèves. On comprend maintenant que les suites de longues le dérangent: contrairement à la quantité prosodique, l'accent tonique est soumis à des règles d'alternance et d'effacement qui lui interdisent de frapper deux syllabes consécutives. En adoptant la proposition de Lote, Baif aurait dû dire adieu aux très nombreux mètres qui admettent deux longues consécutives.

Matthieu Augé-Chiquet adopte une position qui, tout en étant voisine, diffère sensiblement de celle de Lote : 
Sans le savoir, - mais n'est-ce pas ainsi que l'on a fait mainte découverte ? - Baïf inaugurait donc une nouvelle forme de poésie rythmée. On y eût mesuré les vers, non par le nombre des syllabes, mais par celui des ictus rythmiques, et ces ictus, coïncidant avec les accents toniques, eussent été distribués en des proportions et selon des lois à déterminer ${ }^{59}$.

Plutôt que d'établir une correspondance entre les syllabes toniques et les positions longues du schéma métrique, cet auteur préconise de les faire coïncider avec les ictus, autrement dit avec la "battue $"^{60}$ de chaque pied. Plusieurs traducteurs des anciens ont, depuis lors, mis ce programme en pratique :

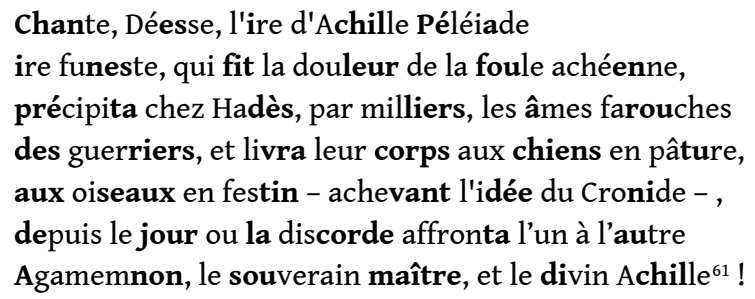

À condition d'admettre qu'ils peuvent frapper tantôt une syllabe tonique, tantôt un " contre-accent " (péléïade, précipita, divin Achille), tantôt un clitique en début de groupe (des, aux, la), on perçoit aisément six ictus par vers, toujours séparés par une ou deux syllabes non marquées. On ne discerne, par contre, aucune différenciation prosodique entre les syllabes «brèves ", celles qui devraient occuper les deux positions brèves du dactyle et les "longues", celles qui devraient figurer le demi-pied ascendant du spondée. Cette prosodie aléatoire, alliée à l'omniprésence des vers féminins, n'est pas sans évoquer les strophes saphiques de Buttet.

On a donc bien une forme de poésie rythmée telle que l'entrevoyait Augé-Chiquet. Baïf aurait-il pu, de son temps, s'en satisfaire ? Il était un prosodiste trop exigeant et trop intimement attaché au modèle antique pour cela.

\section{Une duplicité métrique}

Morin allie une description de la langue de Baiff dont la rigueur est irréprochable avec une analyse de sa métrique qui pose plus de questions qu'elle n'en résout ${ }^{62}$. Pour lui, Baïf serait une espèce d'agent double : affichant, par le biais de sa graphie, une fidélité de façade à la métrique antique, il travaillerait en sous-main à une autre métrique, toute personnelle, dont le principe serait mixte, accentuel et quantitatif. Sur la base de l'examen statistique de 1300 hexamètres dactyliques, Morin discerne, à côté de positions fondées sur la classique opposition longue/brève, des positions "accentuées" dans lesquelles ce serait la présence d'un accent tonique qui serait déterminante. Le schéma métrique ${ }^{63}$ qui résulte d'un tel point de vue n'est adapté, en gros, qu'à deux tiers des vers réels, ce qui rend nécessaire l'élaboration d'un schéma secondaire dont les derniers pieds sont décalés par rapport au schéma principal. Le caractère peu épuré de cette description incline à ne pas la considérer comme définitive.

Selon Morin, Baïf aurait en premier lieu cherché à « reproduire l'impression graphique de la poésie classique $»^{64}$. Sa poésie mesurée aurait donc un aspect pour ainsi dire calligrammatique: la ressemblance superficielle de sa graphie avec le grec, les

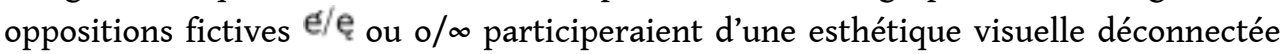
de toute représentation sonore ou rythmique. On a pourtant vu, à propos des vers de 
Jodelle, que cette graphie n'a rien de nécessaire et que les vers peuvent fonctionner sans elle.

On ne sait bien sûr pas avec certitude comment Baïf prononçait le grec et le latin et si ce qui semble assez probable - il adaptait sa prononciation ordinaire de ces langues lorsqu'il scandait des vers, mais on peut se remémorer ces quelques octosyllabes si souvent cités :

L'Iambe dru ie sçay rebatre,

Redoublant le pas qu'il faut batre,

En temps \& lieu, sans fourvoyer :

L'Anapeste ie sçay conduire,

Egaler la demarche : \& duire

Le Chore qu'il faut convoyer.

Ie sçay d'vne assiete acordee

Balansant le pesant Spondee,

Le legier Dactile ranger.

Ie conoy la longue \& la bréve :

Si l'accent baisse ou se relève.

Le François ne m'est étranger ${ }^{65}$.

Peut-on imaginer moins «graphique » que ce programme? Ce n'est pas pour le plaisir de " voir » la longueur par position ou l'accent circonflexe que Baïf apprécie la graphie du grec, mais c'est parce que celle-ci suscite en lui un rythme qu'il peut immédiatement battre et rebattre, duire et conduire, marcher, balancer ou danser, et qui est celui du mètre antique. Aussi, lorsqu'il crée, pour le français, une «imitation » de la graphie grecque dont la prosodie apparente est certes en partie fictive, comprend-on que ce n'est en rien pour « coller » visuellement au modèle. Ce à quoi vise Baiff, c'est à susciter, chez son lecteur, le même éveil rythmique que provoquent en lui les mètres grecs, et à mettre ce rythme métrique répétitif en résonance avec celui, toujours changeant, du discours. Autrement dit, si Baïf, qui est né avec le rythme chevillé au corps, déguise quelque peu sa graphie, ce n'est nullement par duplicité, mais c'est au contraire « pour la bonne cause ", pour la mettre au service du mètre antique « officiel », le seul qu'il ait jamais ressenti dans sa chair.

\section{Une métrique quantitative}

77 Et si, tout bien pesé, Baiff n'avait fait que ce qu'il prétendit faire : composer des vers quantitatifs modernes sur des schémas métriques antiques en s'appuyant sur la prosodie de sa langue paternelle? Il s'agit d'une l'hypothèse à la fois simple et forte, jusqu'ici écartée, pour des raisons diverses et souvent contradictoires, par la longue procession des critiques, mais qui pourrait résister mieux qu'on ne le pense à l'observation des faits.

Porte d'entrée incontournable pour l'étude de ses vers mesurés, la graphie de Baïf n'est pas loin d'avoir livré ses secrets. On sait aujourd'hui, en gros, en démêler les différentes composantes, phonétique, prosodique et métrique et, surtout, en reconnaître les utiles fictions.

La langue écrite, et à plus forte raison la langue poétique, ne correspond jamais exactement à la langue des linguistes: elle n'en a pas la spontanéité, la diversité sociolectale ou dialectale et elle n'évolue pas au même train. Pour cette raison, aucune poésie littéraire ne peut s'appuyer de manière exclusive et immédiate sur une prosodie "proprement linguistique ${ }^{66}$. Inévitablement s'installent des décalages qui nécessitent 
adaptations, conventions et artifices. C'est à ce prix qu'il est possible d'assurer l'interface entre la langue et le mètre. La prosodie de Baïf doit être étudiée pour cela aussi. Fondée certes sur celle de sa langue, elle ne s'y limite pas: la langue fournit l'étalon et les bornes, il appartient à l'homme Baïf de mettre en œuvre le tri. Dans cette opération, il ne peut être réduit au rôle de locuteur naif, jouet d'une phonologie qu'il ne serait pas capable de formaliser ; il est aussi un créateur, doté d'un libre arbitre et d'une responsabilité et qui devra, coûte que coûte, négocier des compromis avec sa langue.

Le corpus est suffisamment volumineux pour qu'une approche statistique soit possible. Lorsque, plusieurs dizaines de fois de suite, Baïf répète le même choix particulier, il faut admettre que, quelles qu'aient été ses raisons, il avait tranché pour le cas général. Si son choix varie d'une occurrence à l'autre, on peut penser qu'il hésitait et n'avait pu se décider. La classe des syllabes communes n'a peut-être pas de fondement linguistique, mais elle découle inéluctablement de la nature même du tri et de celle de l'être humain qui le conduit. Il ne serait pas justifiable de la négliger. Les quelques exemples donnés ici devraient ouvrir la voie à une étude statistique plus complète des choix prosodiques de Baïf.

81 Y a-t-il une métrique particulière à Baïf ? Si oui, peut-elle raisonnablement être autre chose que la somme, ou le résumé, de tout ce qu'il savait de la métrique antique ? Du corpus, on dégage sans peine les schémas métriques, avec leurs alternances de longues et de brèves ainsi que le jeu des substitutions possibles. On sait souvent, parce que, tant dans les Etrénes que dans le psautier A, Baïf a dénommé les vers, quel découpage en pieds il applique. On peut donc faire des conjectures sur la place des ictus rythmiques. Il faudra encore examiner, mètre par mètre, quelles sont les césures auxquelles il recourt et s'il obéit à certaines contraintes ponctuelles ou " ponts ".

En 1981, Reginald Hyatte ${ }^{67}$ relevait déjà que, dans les vers de Baïf, les accents toniques semblent se concentrer sur certaines positions. Les remarques de Bullock vont, on l'a $\mathrm{vu}$, dans le même sens. Sa propre méthode permet à Morin $^{68}$ de donner, pour chaque position de l'hexamètre, un taux de syllabes toniques et, là aussi, il met en évidence d'importantes disparités. De telles observations sont importantes car la succession des accents en français est, on l'admet, rythmogène. Parallèlement au rythme métrique, dicté par les ictus du schéma quantitatif, un autre rythme, qu'on peut qualifier d'accentuel, est susceptible de se manifester. Ce rythme, et c'est là que réside son intérêt, va interagir avec le rythme métrique en le renforçant ou, au contraire, en le contrecarrant, mais sans jamais s'y substituer. Les pics accentuels observés découlentt-ils de l'interaction mécanique d'un schéma métrique donné avec le produit du tri prosodique préalable de Baïf - on a vu que l'accent tonique y jouait son rôle - ou alors procèdent-ils, au moins en partie, d'une intention spécifique du poète? Seule une modélisation statistique poussée, appliquée à un grand nombre de mètres différents, devrait permettre, à l'arrivée, d'en décider ${ }^{69}$. Quoi qu'il en soit, d'éventuels effets rythmiques, s'ils pouvaient être mis en évidence, ne remettraient nullement en question le caractère essentiellement quantitatif de la métrique de Baïf : le fait qu'on rencontre, chez Racine ${ }^{70}$, plus d'accents toniques sur la troisième syllabe du second hémistiche que ce à quoi l'on s'attendrait n'autorise pas pour autant à modifier en conséquence le schéma de l'alexandrin syllabique et à qualifier sa métrique d'accentuelle. 


\section{Reveci venir du printemps}

83

\section{quelques pistes pratiques : \\ REVESI věnir dŭ prīntãns \\ L'ămơrēz' ě bẹlě sẹzōn.}

84 Ces deux vers rendus célèbres par l'œuvre de Claude Le Jeune peuvent être lus comme des octosyllabes, mais Baï a pris soin de noter explicitement le schéma métrique, signalant sans ambiguïté possible un mètre anacréontique. Ce vers est, dans son principe, un dimètre d'ioniques du mineur, avec des ictus ( ,) tombant sur la première longue de chaque pied (positions 3 et 7) :

\begin{tabular}{|c|c|c|c|c|c|c|}
\hline & & ' & & & & , \\
\hline U & $u$ & - & - & $u$ & $u$ & - \\
\hline 1 & 2 & 3 & 4 & 5 & 6 & 7 \\
\hline
\end{tabular}

Dans le cas particulier, le dimètre est en plus modifié par une anaclase (Baïf parlerait d'un vers rebrisé), consistant en l'interversion de deux positions à la jonction des deux pieds (4 et 5), avec pour résultat un effet rythmique analogue à celui de la syncope en musique :

$$
\begin{array}{lllllllllll}
u & u & - & u & 1 & - & u & - & - \\
1 & 2 & 3 & 4 & 5 & 6 & 7 & 8
\end{array}
$$

ce qui, musicalement, équivaut à :

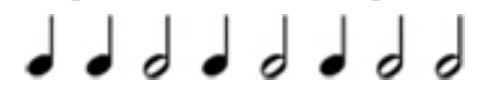

L'interaction de la métrique et de la prosodie provoquera automatiquement la concentration des accents toniques sur certaines positions qu'il est possible, au vu de la simplicité du schéma, de prévoir. On sait que, comme les syllabes féminines sont exclues des positions longues, la dernière position du vers attirera $100 \%$ de syllabes toniques. Comme, en français, il est très rare que deux syllabes consécutives soient accentuées, on en déduit que le taux d'accents toniques sera faible en position 7. Il en ira de même en position 6 , car les positions brèves font en quelque sorte fuir les accents toniques. On s'attend, par compensation, à un important pic accentuel en position 5 , puis à un défaut d'accents dans les positions 4,2 et 1 qui sont brèves, la position 3 restant seule candidate pour accueillir un troisième pic accentuel. Si l'on note les pics accentuels par un !, on obtient le schéma suivant, dans lequel seule la position 3 voit coïncider l'ictus métrique avec un pic accentuel :

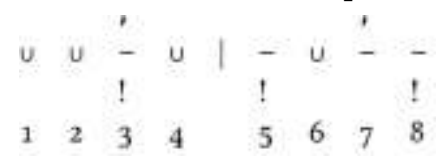

87 Cette dissociation des deux rythmes métrique et accentuel, s'ajoutant à l'effet quasi chorégraphique de l'anaclase, crée une impression de déhanchement perpétuel qui se trouve parfaitement en accord avec l'éthos anacréontique.

Les quatre positions brèves du premier vers coïncident avec trois $e$ féminins et le déterminant $d u$, celles du second vers aux syllabes prétoniques d'amoureux, à la conjonction et et à une syllabe féminine. On ne devrait pas avoir trop de peine à les passer légèrement. La première position longue (reveci) héberge une syllabe qui ne l'est pas particulièrement, mais qui est manifestement accentuée et sur laquelle on 
pourra donc d'appuyer sans retenue. Ensuite, la finale de l'infinitif venir, outre qu'elle est aussi accentuée, offre une consonne implosive avec laquelle il est possible de jouer. Printemps occupe deux positions longues consécutives dont la première est frappée de l'ictus alors que c'est sa deuxième syllabe qui est accentuée. Les deux voyelles sont nasales et, suivant la prononciation adoptée, on devrait pouvoir s'en servir pour les prolonger à volonté. Il faudra peut-être jouer avec l'intonation si l'on veut faire ressortir l'accent tonique, qui ne coïncide pas avec l'ictus. Dans le second vers, la syllabe tonique d'amoureuse se prêtera facilement à la position longue. Graphiquement, les premières syllabes de belle et de saison ne se distinguent pas. Par contre, la première de saison est phonologiquement longue (elle résulte de la simplification d'une diphtongue) alors que la première de belle est juste commune. Il devrait être possible de rendre cette différence par un effet de legato sur saison et de relatif staccato sur belle. La dernière syllabe, -son, quoique tonique et nasale, ne peut pas, comme celle de printemps, faire valoir l's final pour justifier sa longueur. Elle devrait de plus paraître brève en comparaison de la syllabe précédente. La longueur métrique devra donc probablement se reporter sur le silence de l'entrevers.

De la graphie à la diction, en passant par la phonétique, la phonologie, la prosodie la métrique et la musique, les vers mesurés de Baïf ont ceci de particulier qu'ils poussent le vernaculaire français dans ses ultimes retranchements. Au bord de la rupture, celuici laisse alors échapper des secrets que, d'ordinaire, il protège jalousement. Constamment sur le fil qui sépare construction et déconstruction, cette poésie extraordinaire, humaniste jusqu'à l'excès, tout en nourrissant le débat idéologique sur la langue, occupe une place centrale dans l'esthétique du second XVI ${ }^{\mathrm{e}}$ siècle.

\section{BIBLIOGRAPHIE}

AUBIGNÉ, Agrippa de, Euvres, Paris, Lemerre, 1873-1892.

AUGÉ-CHIQUET, Mathieu, La vie, les idées et l'œuvre de Jean-Antoine de Baïf, Hachette, Paris, 1909.

BAÏF, Jean-Antoine de, CEuvres, éd. Marty-Lavaux, Paris, Lemerre, 1882-1890.

BAÏF, Jean-Antoine de, Euvre en vers mesurés, édition électronique par Olivier Bettens, <http://

virga.org/baif/>

BEAUnIER, André, « Parnassiens et Symbolistes », in Le Parnasse, éd. Yann Mortelette, Paris, PUPS, 2006.

BETTENS, Olivier, Chantez-vous français ? <http://virga.org/cvf/>

BETTENS, Olivier, « Récitatif et diction théâtrale : que nous chante l'alexandrin de Quinault-

Lully ? », communication à l'Atelier international sur la prononciation du français vers 1700, Poitiers, nov. 2010, in Chantez-vous français, http://virga.org/cvf/alexanql.php

BETTENS, Olivier, « Octosyllabes, vers mesurés et effets de rythmes », in Jean-Charles MONFERRAN, L'expérience du vers en France à La Renaissance, 2013, p. 185-213. 
BÈZE, Théodore (de), De Francicæe linguce recta pronuntiatione, Genève, 1584.

BONNIFFET, Pierre, « Traces de chant dans les écrits profanes en France avant et après 1570 », Nouvelle revue du seizième siècle, $\mathrm{n}^{\circ}$ 4, p. 5-33.

BULLOCK, Barbara E., « Quantitative verse in a Quantity-Insensitive language : Baif's vers mesurés ", French Language Studies, n 7, 1997, p. 23-45.

BRUNEL, Jean, « La Poésie mesurée française après Jean-Antoine de Baïf », in Claude Le Jeune et son temps en France et dans les états de Savoie, éd. Marie-Thérèse Bouquet-Boyer et Pierre Bonniffet, Berne, Peter Lang, 1996, p. 264-278.

BUTTET, Marc-Claude (de), Le Premier livre des vers, Paris, 1561.

DU BELLAY, Joachim, La deffence et illustration de la langue françoyse, Paris, 1549.

DUBoIs, Claude-Gilbert, La Poésie du XVI e siècle, Bordeaux, Presses universitaires, 1999.

DROZ, Eugénie, « Salomon Certon et ses amis », Humanisme et Renaissance, n VI , 1939, p. 179-197.

HOMÈre, L'Iliade, trad. Philippe Brunet, Paris, Seuil, 2010.

HYATTE, Reginald, « Meter and Rhytm in Jean-Antoine de Baï's Étrénes de poézie fransoèze and the vers mesurés à l'antique of Other Poets in the Late Sixteenth Cenrury », Bibliothèque d'Humanisme et de Renaissance, ${ }^{\circ}$ 43, 1981, p. 487-508.

JODELLE, Étienne, CEuvres, Paris, 1574.

LA RAMÉE, dit Ramus, Pierre (de), Grammaire, Paris, 1572.

LA TAILlE, Jacques (de), La Maniere de faire des vers en françois, comme en grec \& en latin, Paris, 1573.

LOTE, Georges, Histoire du vers français, Paris, Boivin, puis Hatier, puis Aix, Université de Provence, 1949-1996.

MORIN, Yves-Charles, « La Graphie de Jean-Antoine de Baïf : au service du mètre », Nouvelle Revue du Seizième Siècle, $n^{\circ}$ 17(1), 1999, p. 85-106.

MORIN, Yves-Charles, "L'Hexamètre "héroïque" de Jean-Antoine de Baïf », in Métriques du Moyen Age et de la Renaissance, éd. Dominique Billy, Paris, L'Harmattan, 1999, p. 163-184.

MORIN, Yves-Charles, « La Prononciation et la prosodie du français du XVI ${ }^{\mathrm{e}}$ siècle selon le témoignage de Jean-Antoine de Baï », Langue française, $\mathrm{n}^{\circ}$ 126, 2000, p. 9-28.

NOUGARET, Louis, Traité de métrique latine classique, Paris, Klincksieck, 1986.

PASQUIER, Étienne, Les Recherches de la France, Paris, 1621.

TOUTAIN, Charles, La Tragedie d'Agamemnon, Paris, 1556.

VIGNES, Jean, « Brève histoire du vers mesuré français au XVI ${ }^{\mathrm{e}}$ siècle », Albineana, $\mathrm{n}^{\circ}$ 17, 2005, p. $15-43$.

WEBER, Édith, «Le Prototype de la Strophe sapphique : son exploitation musicale au XVI siècle », in Claude Le Jeune et son temps en France et dans les états de Savoie, éd. Marie-Thérèse Bouquet-Boyer et Pierre Bonniffet, Berne, Peter Lang, 1996, p. 16-34.

\section{NOTES}

1. Joachim Du Bellay, La Deffence, II-vii. 
2. Parfois aussi graphié omiotelefte, ce qui donne une idée de la variété des prononciations du grec qui pouvait exister chez les humanistes. Cf. Étienne Pasquier, Recherches, p. 595.

3. Joachim Du Bellay, Deffence, I-ix.

4. Ibid., II-viii.

5. Étienne Jodelle, Euvres (1574), préface posthume de Charles de La Mothe.

6. Jacques de La Taille, La Maniere, p. 2 ro.

7. Louis Nougaret, Traité de métrique latine classique, p. 106.

8. Imprimée en 1561, elle pourrait néanmoins avoir été composée avant les vers dont Étienne Pasquier, Recherches, p. 650, crédite Jodelle (1553), Denisot (1555) et lui-même (1556). On ne fera pas état ici du travail de Boteauville (avant 1500), qui apparaît complètement détaché de ce mouvement.

9. Marc-Claude de Buttet, Le Premier Livre, p. $45 \mathrm{r}^{\circ}$.

10. Étienne Pasquier, Recherches, p. 652.

11. Ibid., p. 624.

12. Par convention, on parle de césure "lyrique » lorsqu'une syllabe féminine numéraire et non élidée apparaît à la césure.

13. Pierre de La Ramée, dit Ramus, Grammaire, p. 43-44.

14. Voir à ce propos Olivier Bettens, "Chronique d'un éveil prosodique", in Chantez-vous français? <http://virga.org/cvf/chronique.php>

15. Le lecteur est renvoyé une fois pour toute à mon édition en ligne des vers mesurés de Baïf, disponible sur <http://virga.org/baif>. Elle offre, sous « Dictionnaire », un outil de recherche par mots ou chaînes de caractères grâce auquel on pourra sans difficulté localiser les exemples cités ici.

16. Selon Jean Vignes, «Brève histoire... », p. 38, qui s'appuie sur les chiffres fournis par Jean Brunel.

17. Jean Brunel, «La Poésie mesurée française après Jean Antoine de Baïf », p. 266.

18. L'estimation de Brunel, soit environ 70, est grossièrement en dessous de la réalité. Le répertoire constitué pour mon édition des vers mesurés de Baïf recense près de 110 mètres pour lesquels on pourrait formuler une dénomination spécifique, certains d'entre eux donnant en plus lieu à de nombreuses variantes.

19. Il en résulte que les mots en -ura sont paroxytons (accent tonique sur la pénultième syllabe) alors que ceux en -ula sont proparoxytons (accent tonique sur l'antépénultième).

20. Jacques de La Taille, La Maniere, p. $9 \mathrm{r}^{\circ}$.

21. Théodore de Bèze, De Francica linguce, p. 75-76.

22. Voir à ce propos Yves-Charles Morin, «La graphie de Jean-Antoine de Baiff... » et «La prononciation et la prosodie... ».

23. Jacques de La Taille, La Maniere, p. $6 \mathrm{v}^{\circ}$.

24. Arma virumque cano: dans cet exemple, les syllabes fermées, et donc longues par position ont été soulignées. On se gardera de confondre la longueur par "position", qui est une notion prosodique avec les "positions» métriques, qui constituent les cases des schémas abstraits décrivant les vers.

25. Il ne faut pas confondre position indifférente avec syllabe commune. Dans le premier cas, on a une catégorie métrique qui tolère toutes les valeurs prosodiques; dans le second, on a une catégorie prosodique qui peut s'adapter à toutes les valeurs métriques.

26. Cette épithète désigne, quoique de manière floue, les nom, les adjectifs, les formes verbales et les adverbes. Par opposition, les «clitiques» sont des mots outils (articles, pronoms, prépositions, etc.) qui n'ont pas d'accent propre mais s'associent à un mot " plein » pour former un groupe.

27. Jacques de La Taille, La Maniere, p. 9 v. 
28. Ibid., p. $10 \mathrm{r}^{\circ}$. Les syllabes marquées ici d'un * sont, dans l'impression originale, coiffées d'un micron et d'un macron superposés.

29. Ibid., p. $7 \mathrm{r}^{\circ}$.

30. Yves-Charles Morin, «La prononciation et la prosodie... », p. 12. La distinction, dans un corpus écrit comme celui de Baï, entre syllabe (potentiellement) accentuable et syllabe (effecivement) accentuée comporte un gros risque de circularité. Est-ce parce qu'elle est accentuée qu'une syllabe se trouvera scandée longue ou parce qu'elle est scandée longue qu'on la qualifiera d'accentuée?

31. Salomon Certon, Traité sommaire de la quantité Françoise, manuscrit inédit cité par Eugénie DROZ, « Salomon Certon et ses amis ».

32. Étienne Jodelle, Euvres, p. 115.

33. Charles Toutain, Chant IX. Merci à Olivier Halévy qui m'en a fourni sa copie. Baïf aurait vraisemblablement fait brève la première syllabe de avertir et longue la dernière de viendra.

34. Yves-Charles Morin, « La Graphie de Jean-Antoine de Baïf... ».

35. Jean-Antoine de Baïf, "Aux secrétaires d'État », Etrénes. <http://virga.org/baif/index.php? item=167>

36. Pour un tableau synoptique du système graphique de Baif, voir l'introduction de mon édition. $<$ http://virga.org/baif/index.php?item=98>

37. Théodore de Bèze, De Francica linguce, p. 57 et sq.

38. Voir à ce propos Olivier Bettens, «Les consonnes finales ", in Chantez-vous français. <http:// virga.org/cvf/consfina.php>

39. En première approximation et faute d'un meilleur critère, on admettra qu'une syllabe (ou une catégorie de syllabes) est commune si son nombre d'occurrences en position brève et son nombre d'occurrences en position longue sont tous deux supérieurs à $5 \%$ du total.

40. Pour ce comptage, et ceux qui suivent, on n'a retenu que les voyelles graphiquement « communes », c'est-à-dire celles dont la valeur métrique n'est pas déductible de la graphie.Ę, $\infty$, o, e ainsi que les «diphtongues» et les voyelles surmontées d'un accent circonflexe ont été exclus.

41. La prosodie latine connaît un effet abrégeant de la consonne finale -t sur la voyelle qui précède. Voir Louis Nougaret, Traité de métrique latine classique, p. 49.

42. Jean-Antoine de Baïf, Introduction des Etrénes, <http://virga.org/baif/index.php?item=187>.

43. Yves-Charles Morin, «La prononciation et la prosodie... ", p. 13.

44. On note la même attitude pour la conjonction car dont l'étymologie (quāre) ressemble à celle des infinitifs en -er (-äre) : longue par position devant consonne initiale et presque toujours brève devant voyelle.

45. Contrairement à ce qu'affirme Morin sur la base de son échantillon, "L'Hexamètre héroïque... ", p. 179, il est tout sauf évident que, dans l'ensemble du corpus, père apparaisse dans une position brève lorsqu'on attend une forme inaccentuée comme dans l'expression figée père et mère. On a par exemple de pęr' an fis et De pęr' é d'onkl' (position longue) en face de le Pér' an se mokant rit (le père, en se moquant, rit, position brève) où père aurait toutes les raison d'être accentué.

46. Claude-Gilbert Dubois, La Poésie du XVIe siècle, p. 101.

47. Dubois scande Phēbūs / Āmoūr, / Cȳprīs / veūt saŭvěr / noūrrŭr ĕt / ōrnēr alors que Jodelle entend évidemment Phēbǔs ă / moūr, Cȳ| prīs veūt / saūvēr / noūrrür ĕt / ōrnēr.

48. André Beaunier, « Parnassiens et symbolistes », p. 317.

49. Agrippa d'Aubigné, CEuvres, I, p. 454.

50. Jean Vignes, « Brève histoire... ", p. 24 et sq.

51. Pierre Bonniffet, "Traces du chant dans les écrits profanes...». Cet article contient en annexe, sous le titre «L'Union de la musique à la poésie selon J.-A. de Baïf », un tableau rudimentaire de son système graphique, p. 22-23. 
52. Barbara E. Bullock, « Quantitative verse... ».

53. Dans le schéma de référence de ce vers, tel que le donne Baïf au début du psautier A de 1569 en séparant les mètres par des virgules ( $\mathrm{X}-\cup_{-}^{-} \mathrm{X}-\cup_{-}^{-} \mathrm{X}-\smile-$ ), les ictus métriques tombent par définition sur les demi-pieds pairs (ou descendants), soit les positions marquées d'un macron. Bullock, en insérant la césure après le cinquième demi-pied, transforme cette position indifférente en une position longue. Cette procédure ad hoc fait apparaitre comme faussement irréguliers les vers dont ce cinquième demi-pied est occupé par une syllabe féminine ; elle permet surtout à Bullock de fermer les yeux sur le fait que, chez Baïf, les syllabes toniques tendent à se concentrer sur ce demi-pied en dépit du fait qu'il est ascendant et donc dépourvu d'ictus. Avant elle, Hyatte, « Meter and Rhythm... », avait, du point de vue de l'accent, analysé le début de ce mètre comme « un iambe suivi d'un anapeste ». Comme Bullock, il avait considéré ce mètre comme isosyllabique alors même que, sur l'ensemble du corpus, Baïf recourt assez couramment à des substitutions qui en font varier le nombre de syllabes.

54. Édith Weber, « Le prototype de la strophe sapphique », p. 28.

55. Yves-Charles Morin, « La prononciation et la prosodie... », p. 26.

56. Voir à ce propos Olivier Bettens, «Les consonnes finales - Les raisons de la rime », in Chantezvous français. <http://virga.org/cvf/consfina.php\#raisonrime>

57. Georges Lote, Histoire du Vers français, IV, p. 150.

58. Georges Lote, Histoire du Vers français, IV, p. 152.

59. Mathieu Augé-Chiquet, La Vie, les idées et l'œuvre de Jean-Antoine de Baï, p. 353.

60. Selon l'époque et le système de référence on peut aussi parler de thesis, de tactus, de touchement, de posé, de temps frappé, de downbeat etc. Tous ces termes sont équivalents et désignent l'événement saillant dont la récurrence caractérise tout rythme.

61. Homère, début de l'Iliade, dans la belle traduction de Philippe Brunet, parue en 2010. Dans cet extrait, les syllabes frappées par l'ictus ont été mises en évidence.

62. Yves-Charles Morin, «L'hexamètre "héroïque"... ».

63. Le premier des deux schémas fournis par Morin pour l'hexamètre dactylique se décline ainsi :

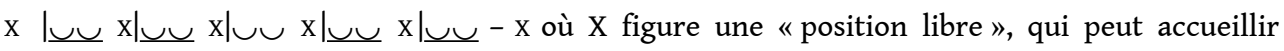
n'importe quelle syllabe, $x$ une position accentuée pouvant être remplie par une syllabe «de poids [durée] quelconque (de préférence tonique) » et où $u$ peut accueillir alternativement une syllabe longue ou deux brèves. On note, par rapport au schéma classique (six dactyles avec substitution possible par des spondées), une étrange inversion du découpage en pieds. On s'interroge aussi sur la pertinence de la catégorie "accentuée » dont la "préférence " pour les syllabes toniques est loin d'être absolue. Oserait-on qualifier une position de «brève " si elle ne faisait que préférer les syllabes brèves tout en tolérant quelques longues?

64. Yves-Charles Morin, « La Graphie... », p. 100.

65. Jean-Antoine de Baif, dédicace des Jeux, éd. Marty-Laveaux, III, p. 3.

66. Yves-Charles Morin, «L'Hexamètre héroïque... », p. 163. Un problème de l'approche de Morin est peut-être justement d'avoir cherché à arrimer trop directement la métrique à la phonologie. Comme, dans cette démarche, il n'y a pas de place pour une prosodie adaptée à la métrique, c'est inévitablement la métrique qui en fait les frais avec pour conséquence une dislocation du schéma authentique de l'hexamètre.

67. Reginald Hyatte, « Meter and Rhythm... », p. 494-497.

68. Yves-Charles Morin, «L'hexamètre héroïque... », p. 173 et sq.

69. On trouvera un début de réponse à cette question dans Olivier Bettens, «Octosyllabes, vers mesurés et effets de rythmes ».

70. Olivier Bettens, « Récitatif et diction théâtrale... ». 


\section{RÉSUMÉS}

Le renouveau des vers mesurés est l'un des mouvements les moins bien compris de toute l'histoire littéraire. Après les avoir replacés dans la démarche d'illustration des vernaculaires qui prévaut au $\mathrm{XVI}^{\mathrm{e}}$ siècle, on s'attache à expliciter la démarche de Jean-Antoine de Baïf qui est, de loin, la plus aboutie en la matière.

\section{INDEX}

Index géographique : domaine français

Mots-clés : illustration du français, métrique, prosodie, quantité syllabique, vers mesurés, phonétique, Jean-Antoine de Baïf, académies, poétique, poésie, musique, gaulois

Index chronologique : XVIe siècle

\section{AUTEUR}

\section{OLIVIER BETTENS}

Historien du chant, de la prosodie et de la déclamation, Olivier Bettens est chercheur indépendant. Il a réalisé l'édition en ligne des vers mesurés de Jean-Antoine de Baïf. 